\title{
Sur l'ordre de grandeur des polynômes de Dirichlet
}

\author{
par
}

RÉGis DE LA BRETÈChe (Paris)

1. Introduction et énoncés des résultats. À une suite de nombres complexes $A:=\left\{a_{n}\right\}_{n=1}^{\infty}$, on associe une série de Dirichlet

$$
A(s):=\sum_{n=1}^{\infty} a_{n} n^{-s} .
$$

Considérant sa troncature

$$
A_{x}(s):=\sum_{n \leq x} a_{n} n^{-s}
$$

on note les normes

$$
\begin{aligned}
\left\|A_{x}\right\|_{\infty} & :=\sup _{\tau \in \mathbb{R}}\left|A_{x}(i \tau)\right|=\sup _{\tau \in \mathbb{R}}\left|\sum_{n \leq x} a_{n} n^{-i \tau}\right|, \\
\left\|A_{x}\right\|_{1} & :=\sum_{n \leq x}\left|a_{n}\right|, \quad\left\|A_{x}\right\|_{2}:=\sqrt{\sum_{n \leq x}\left|a_{n}\right|^{2}} .
\end{aligned}
$$

Nous nous proposons dans ce travail de comparer les normes $\left\|A_{x}\right\|_{1}$ et $\left\|A_{x}\right\|_{\infty}$. On définit $S\left(\Lambda_{x}\right)$, la constante de Sidon associée à l'ensemble $\Lambda_{x}:=$ $\{\log n: n \leq x\}\left(^{1}\right)$ comme le plus petit réel $C_{x}$ tel que l'on ait pour toute suite $A$ l'inégalité

$$
\left\|A_{x}\right\|_{1} \leq C_{x}\left\|A_{x}\right\|_{\infty}
$$

Soit

$$
L(x):=\exp \sqrt{\log x \log _{2} x},
$$

où $\log _{2}$ désigne, ici et par la suite, la deuxième itérée du logarithme. Dans [5] et [4] (voir aussi [1]), Konyagin et Queffélec ont montré qu'il existe une

2000 Mathematics Subject Classification: 11M41, 11N37, 42A05.

Key words and phrases: Dirichlet series, friable integers, Sidon sets, Berstein's inequality, Salem-Zygmund's inequality, Bohr's inequality.

$\left({ }^{1}\right)$ De manière générale, on définit $S(\Lambda)$, la constante de Sidon associée à un ensemble $\Lambda$, comme le plus petit réel $C$ tel que pour toute suite $A$ on ait $\sum_{\ell \in \Lambda}\left|a_{\ell}\right| \leq$ $C \sup _{\tau \in \mathbb{R}}\left|\sum_{\ell \in \Lambda} a_{\ell} e^{i \ell \tau}\right|$. 
constante $\beta>0$ telle que lorsque $x$ tend vers l'infini,

$$
\sqrt{x} L(x)^{-\sqrt{2}+o(1)} \leq S\left(\Lambda_{x}\right) \leq \sqrt{x} L(x)^{-\beta+o(1)} .
$$

Notre résultat est le suivant.

ThÉORÈme 1.1. Lorsque $x$ tend vers l'infini, on a l'estimation

$$
\sqrt{x} L(x)^{-\sqrt{2} / 2+o(1)} \leq S\left(\Lambda_{x}\right) \leq \sqrt{x} L(x)^{-\sqrt{2} / 4+o(1)} .
$$

Une version effective des $o$ découle directement des méthodes employées.

C'est un plaisir de remercier Hervé Queffélec de son aide lors de la rédaction de cet article.

\section{Démonstrations}

\subsection{Minoration de $S\left(\Lambda_{x}\right)$}

Lemme 2.1. Lorsque $x$ tend vers l'infini, on a l'estimation

$$
S\left(\Lambda_{x}\right) \geq \sqrt{x} L(x)^{-\sqrt{2} / 2+o(1)} .
$$

Démonstration. On reprend la méthode de Queffélec [5] qui utilise les entiers $y$-friables. Soit $P^{+}(n)$ le plus grand facteur premier d'un entier générique $n$ avec la convention $P^{+}(1)=1$. On dit qu'un entier $n$ est $y$-friable si $P^{+}(n) \leq y$. Conformément à l'usage, nous notons

$$
S(x, y):=\left\{n \leq x: P^{+}(n) \leq y\right\}, \quad \Psi(x, y):=|S(x, y)|,
$$

et $\mathbf{1}_{S(\infty, y)}$ la fonction caractéristique des entiers $y$-friables.

On considère la suite $A:=\left\{a_{n}\right\}_{n=1}^{\infty}$ définie par $a_{n}:=\varepsilon_{n} \mathbf{1}_{S(\infty, y)}(n)$ où $\left\{\varepsilon_{n}\right\}_{n=1}^{\infty}$ est une suite de Rademacher prenant \pm 1 comme valeur $\left({ }^{2}\right)$. L'ordre de la quantité $\left\|A_{x}\right\|_{1}=\Psi(x, y)$ est bien connue (cf. le livre de Tenenbaum [6, Chapitre III.5]). Conformément à l'usage, nous notons $\varrho(v)$ la fonction de Dickman, définie comme l'unique solution continue à droite sur $\mathbb{R}$ tout entier de l'équation différentielle aux différences

$$
v \varrho^{\prime}(v)+\varrho(v-1)=0 \quad(v>1)
$$

avec les conditions initiales $\varrho(v)=0(v<0)$ et $\varrho(v)=1(0 \leq v \leq 1)$. Posant $u=\log x / \log y$, on utilisera l'estimation uniforme

$$
\Psi(x, y) \sim x \varrho(u)
$$

dans le domaine

$$
\exp \left\{\left(\log _{2} x\right)^{5 / 3+\varepsilon}\right\} \leq y \leq x, \quad x \geq 2,
$$

$\left({ }^{2}\right)$ Une suite de Rademacher est une suite de variables aléatoires indépendantes sur un espace de probabilité $(\Omega, \mathcal{A}, P)$ avec $P\left(\varepsilon_{n}=1\right)=P\left(\varepsilon_{n}=-1\right)=1 / 2$. On pourra se reporter au livre de Kahane [3] où sont détaillées de nombreuses exemples de l'utilisation de ces suites. 
relatif à un réel $\varepsilon>0$ fixé. La seule information sur la fonction $\varrho$ dont on aura besoin ici est l'estimation

$$
\varrho(u)=\exp \left\{-u\left(\log u+\log _{2}(2 u)+O(1)\right)\right\} \quad(u \geq 1) .
$$

Soit $\Gamma$ le cercle unité du plan complexe. La densité de

$$
\left\{\left(e^{2 \pi i \tau \log p_{1}}, \ldots, e^{2 \pi i \tau \log p_{k}}\right): \tau \in \mathbb{R}\right\}
$$

dans $\Gamma^{k}$ permet d'affirmer que

$$
\left\|A_{x}\right\|_{\infty}=\sup _{\mathbf{t} \in[0,1]^{\pi(y)}}\left|P_{x, y}\left(e^{2 \pi i t_{1}}, \ldots, e^{2 \pi i t_{\pi(y)}}\right)\right|
$$

où $P_{x, y}(\mathbf{z})$ désigne le polynôme en $\pi(y)$ variables défini par

$$
P_{x, y}(\mathbf{z}):=\sum_{n \in S(x, y)} \varepsilon_{n} z_{1}^{v_{p_{1}}(n)} \ldots z_{\pi(y)}^{v_{p_{(y)}}(n)}
$$

avec $\left\{p_{i}\right\}_{i=1}^{\infty}$ la suite croissante des nombres premiers et $v_{p}$ la valuation $p$-adique. Posons

$$
Q_{x, y}(\mathbf{t}):=P_{x, y}\left(e^{2 \pi i t_{1}}, \ldots, e^{2 \pi i t_{\pi(y)}}\right) .
$$

On a ainsi

$$
\left\|A_{x}\right\|_{\infty}=\left\|Q_{x, y}\right\|_{\infty} \cdot\left({ }^{3}\right)
$$

On a, pour tout $n \in S(x, y)$,

$$
\Omega(n)=v_{p_{1}}(n)+\cdots+v_{p_{\pi(y)}}(n) \leq \log x / \log 2 .
$$

Soit $B$ l'ensemble des fonctions $Q$ de la forme

$$
Q(\mathbf{t})=\sum_{n \in S(x, y)} a_{n} e^{2 i \pi \eta_{1} v_{p_{1}}(n) t_{1}} \cdots e^{2 i \pi \eta_{\pi(y)} v_{p_{\pi(y)}}(n) t_{\pi(y)}}
$$

où

$$
a_{n} \in \mathbb{R}, \quad\left\{\eta_{k}\right\}_{k=1}^{\pi(y)} \in\{-1,1\}^{\pi(y)} .
$$

La présence des $\eta_{i}$ permet de rendre l'ensemble $B$ invariant par conjugaison complexe.

Soit $I$ l'ensemble des t tels que

$$
|Q(\mathbf{t})| \geq \frac{1}{2}\|Q\|_{\infty}
$$

L'inégalité de Bernstein (cf. Queffélec [5, p. 45]) fournit, pour toute fonction $Q \in B$,

$$
\left|Q(\mathbf{t})-Q\left(\mathbf{t}^{\prime}\right)\right| \leq \delta \sup _{k=1, \ldots, \pi(y)}\left|t_{k}-t_{k}^{\prime}\right|\|Q\|_{\infty}
$$

où l'on a posé $\delta:=\pi^{2} \log x / \log 2$. Cela implique que $\mu(I) \geq \delta^{-\pi(y)}$ où $\mu$ est la mesure de Lebesgue de $[0,1]^{\pi(y)}$. En effet, pour un élément $Q \in B$,

$\left({ }^{3}\right)$ Ici, on a utilisé la notation classique de la norme infinie pour deux espaces différents de fonctions. 
il existe un point $\mathbf{t}_{0} \in[0,1]^{\pi(y)}$ satisfaisant $\left|Q\left(\mathbf{t}_{0}\right)\right|=\|Q\|_{\infty}$. Le multi-carré d'arête de $\mathbb{R}^{\pi(y)}$ de longueur $1 / \delta$ centré en un $\mathbf{t}_{0}$ est alors inclus dans $I$.

Nous sommes maintenant en mesure d'appliquer l'inégalité de SalemZygmund (cf. Kahane [3, Theorem VI.1]). Il vient

$$
\mathbb{P}\left(\left\|Q_{x, y}\right\|_{\infty} \geq 3 \sqrt{\Psi(x, y) \log \left(8 \delta^{\pi(y)}\right)}\right) \leq 1 / 2 .
$$

D'après $(2 \cdot 4)$, il existe donc une suite de Rademacher $\left\{\varepsilon_{n}\right\}_{n \leq x}$ telle que

$$
\left\|A_{x}\right\|_{\infty} \ll \sqrt{\Psi(x, y) \pi(y) \log _{2} x} .
$$

Notons qu'une conséquence plus forte que $(2 \cdot 8)$ du Théorème VI.1 de [3] est l'inégalité

$$
E\left(\left\|A_{x}\right\|_{\infty}\right) \ll \sqrt{\Psi(x, y) \pi(y) \log _{2} x} .
$$

Mais l'inégalité $(2 \cdot 8)$ est suffisante pour montrer $(2 \cdot 9)$. De $(2 \cdot 9)$, on déduit que

$$
\left\|A_{x}\right\|_{1}=\Psi(x, y) \leq S\left(\Lambda_{x}\right)\left\|A_{x}\right\|_{\infty} \ll S\left(\Lambda_{x}\right) \sqrt{\Psi(x, y) \pi(y) \log _{2} x} .
$$

Il en découle que

$$
S\left(\Lambda_{x}\right) \geq \sqrt{\frac{\Psi(x, y)}{\pi(y) \log _{2} x}} .
$$

Il reste à choisir la valeur de $y$. On écrit $y=\exp \left\{\alpha \sqrt{\log x \log _{2} x}\right\}$ où $\alpha>0$ est un paramètre à fixer. De $(2 \cdot 1),(2 \cdot 2)$ et du théorème des nombres premiers, il découle

$$
\begin{aligned}
S\left(\Lambda_{x}\right) & \geq \sqrt{\frac{x \log y}{\log _{2} x}} \exp \left(\frac{1}{2} \log \varrho(u)-\frac{\log x}{2 u}\right) \\
& \geq \sqrt{x} \exp \left\{-\left(\frac{1}{4 \alpha}+\frac{\alpha}{2}+o(1)\right) \sqrt{\log x \log _{2} x}\right\} .
\end{aligned}
$$

Le choix optimal $\alpha=1 / \sqrt{2}$ fournit alors le résultat souhaité.

2.2. Majoration de $S\left(\Lambda_{x}\right)$. L'énoncé suivant permet d'obtenir une valeur explicite de $\beta$ dans la majoration (1.1) de $S\left(\Lambda_{x}\right)$ établie par Konyagin et Queffélec [4].

Lemme 2.2. Lorsque $x$ tend vers l'infini, on a l'estimation

$$
S\left(\Lambda_{x}\right) \leq \sqrt{x} L(x)^{-\sqrt{2} / 4+o(1)} .
$$

Démonstration. Nous reprenons la méthode de Konyagin et Queffélec. Afin de la rendre effective, nous utilisons les lemmes suivants.

Soient $P^{-}(n)$ le plus petit facteur premier d'un entier générique $n$ avec la convention $P^{-}(1)=1$, et $\Omega(n)$ la fonction qui compte les facteurs premiers de $n$ avec multiplicité. On introduit les ensembles

$$
T(x, y):=\left\{n \leq x: P^{-}(n)>y\right\}, \quad T_{k}(x, y):=\{n \in T(x, y): \Omega(n)=k\} .
$$


Soit

$$
N_{k}(x, y):=\operatorname{card}\{n \in T(x, y): \Omega(n) \geq k\}=\sum_{j \geq k} \operatorname{card}\left\{T_{j}(x, y)\right\} .
$$

Lemme 2.3. Il existe $c>0$ tel que, pour $x \geq y \geq 2$ et $k \geq 1$, on ait l'estimation uniforme

$$
N_{k}(x, y) \ll \frac{x}{y^{k}}(\log x)^{y-1} e^{c y} .
$$

Ce résultat, remarquable par son uniformité, est dû à Balazard ([2, Corollaire 1]).

Lemme 2.4. Pour toute suite de nombres de complexes $A:=\left\{a_{n}\right\}_{n=1}^{\infty}$, tout entier $k \geq 1$ et $x \geq 2$, on a

$$
\left(\sum_{\substack{n \leq x \\ \Omega(n)=k}}\left|a_{n}\right|^{2 k /(k+1)}\right)^{(k+1) / 2 k} \leq k^{k / 2}\left\|A_{x}\right\|_{\infty} .
$$

Ce résultat est dû à Queffélec [5] (voir aussi [4]). Il peut être vu comme une généralisation de l'inégalité de Bohr qui correspond au cas $k=1$.

On peut maintenant démontrer le Lemme 2.2. La densité de l'ensemble $\left\{\left(e^{2 \pi i t \log p_{1}}, \ldots, e^{2 \pi i t \log p_{k}}\right): t \in \mathbb{R}\right\}$ dans $\Gamma^{k}$ permet d'affirmer que

$$
\left\|A_{x}\right\|_{\infty}=\sup _{\mathbf{z} \in \Gamma^{\pi(x)}}\left|P_{x}(\mathbf{z})\right|
$$

où $P_{x}(\mathbf{z})$ est le polynôme en $\pi(x)$ variables défini par

$$
P_{x}(\mathbf{z}):=\sum_{n \leq x} a_{n} z_{1}^{v_{p_{1}}(n)} \cdots z_{\pi(x)}^{v_{p_{\pi(x)}}(n)} .
$$

Tout entier $n$ se décompose de manière unique sous la forme

$$
n=m \ell, \quad m \in S(\infty, y), \ell \in T(\infty, y) .
$$

On pose $y:=\sqrt{\log x} / \log _{2} x$. Soit $P_{x, y, m}$ le polynôme appartenant à $\mathbb{C}\left[z_{\pi(y)+1}, \ldots, z_{\pi(x)}\right]$ défini par

$$
P_{x, y, m}(\mathbf{z}):=\sum_{\ell \in T(x / m, y)} a_{m \ell} z_{\pi(y)+1}^{v_{p_{\pi(y)+1}}(\ell)} \cdots z_{\pi(x)}^{v_{p_{\pi(x)}(\ell)}}
$$

associé à

$$
A_{x, y, m}(t):=\sum_{\ell \in T(x / m, y)} a_{m \ell} \ell^{i t}
$$


Suivant la remarque de [4] (formule (3.13)), on a

$$
\begin{aligned}
P_{x, y, m}(\mathbf{z}) & =\frac{1}{(2 \pi)^{\pi(y)}} \\
& \times \int_{0}^{2 \pi} \cdots \int_{0}^{2 \pi} P_{x}\left(e^{i \vartheta_{1}}, \ldots, e^{i \vartheta_{\pi(y)}}, \mathbf{z}\right) e^{-i \alpha_{1} \vartheta_{1}-\cdots-i \alpha_{\pi(y)} \vartheta_{\pi(y)}} \mathrm{d} \vartheta_{1} \cdots \mathrm{d} \vartheta_{\pi(y)},
\end{aligned}
$$

avec $m=\prod_{j \leq \pi(y)} p_{j}^{\alpha_{j}}$. On en déduit l'inégalité

$$
\left\|A_{x, y, m}\right\|_{\infty}=\sup _{\mathbf{z} \in \Gamma^{\pi(x)-\pi(y)}}\left|P_{x, y, m}(\mathbf{z})\right| \leq \sup _{\mathbf{z} \in \Gamma^{\pi(x)}}\left|P_{x}(\mathbf{z})\right|=\left\|A_{x}\right\|_{\infty} .
$$

La décomposition $(2 \cdot 10)$ permet d'écrire

$$
\begin{aligned}
\left\|A_{x}\right\|_{1} & =\sum_{m \in S(x, y)} \sum_{\ell \in T(x / m, y)}\left|a_{m \ell}\right| \\
& \leq \Psi(x, y) \sup _{m \in S(x, y)}\left(\sum_{\ell \in T(x / m, y)}\left|a_{m \ell}\right|\right) .
\end{aligned}
$$

Posons $K:=\vartheta \sqrt{\log x / \log _{2} x}$ où le réel $\vartheta>0$ est un paramètre. D'une part, les Lemmes 2.3 et 2.4 fournissent, pour $k \leq K$,

$$
\begin{aligned}
\sum_{\ell \in T_{k}(x / m, y)}\left|a_{m \ell}\right| & \leq\left(\sum_{\ell \in T_{k}(x / m, y)}\left|a_{m \ell}\right|^{2 k /(k+1)}\right)^{(k+1) / 2 k}\left|T_{k}(x, y)\right|^{(k-1) / 2 k} \\
& \leq k^{k / 2}\left\|A_{x, y, m}\right\|_{\infty}\left|T_{k}(x, y)\right|^{(k-1) / 2 k} \\
& \ll k^{k / 2}\left\|A_{x}\right\|_{\infty} \sqrt{x} \frac{x^{-1 / 2 k}}{y^{(k-1) / 2}}(\log x)^{(y-1) / 2} e^{c y / 2} \\
& =: \sqrt{x}\left\|A_{x}\right\|_{\infty} e^{h_{x, y}(k)}
\end{aligned}
$$

où l'on a utilisé l'inégalité de Hölder et la relation $(2 \cdot 11)$. Pour $x$ suffisamment grand et $y=\sqrt{\log x} / \log _{2} x$, la suite $h_{x, y}$ ainsi définie est croissante pour $k \leq K$. Il vient

$$
\sum_{k \leq K} \sum_{\ell \in T_{k}(x / m, y)}\left|a_{m \ell}\right| \ll \sqrt{x}\left\|A_{x}\right\|_{\infty} K e^{h_{x, y}(K)} .
$$

D'autre part, l'inégalité de Cauchy-Schwarz fournit

$$
\sum_{\ell \in \bigcup_{k>K} T_{k}(x / m, y)}\left|a_{m \ell}\right| \leq\left(\sum_{n \leq x}\left|a_{n}\right|^{2}\right)^{1 / 2} N_{K}(x, y)^{1 / 2}=\left\|A_{x}\right\|_{2} N_{K}(x, y)^{1 / 2} .
$$

On fait appel à la majoration

$$
\left\|A_{x}\right\|_{2} \leq\left\|A_{x}\right\|_{\infty}
$$


issue de la majoration

$$
\left\|A_{x}\right\|_{2}^{2}=\int_{[0,1]^{\pi(x)}} \cdots \int_{x}\left|P_{x}\left(e^{2 i \pi t_{1}}, \ldots, e^{2 i \pi t_{\pi(x)}}\right)\right|^{2} \mathrm{~d} t_{1} \cdots \mathrm{d} t_{\pi(x)} \leq\left\|A_{x}\right\|_{\infty}^{2} .
$$

Du Lemme 2.3, on obtient alors

$$
\sum_{\ell \in \bigcup_{k>K} T_{k}(x / m, y)}\left|a_{m \ell}\right| \leq \sqrt{x}\left\|A_{x}\right\|_{\infty}(\log x)^{(y-1) / 2} e^{c y / 2} y^{-K / 2} .
$$

En remarquant que

$$
\Psi(x, y) \leq\left(1+\frac{\log x}{\log 2}\right)^{\pi(y)} \ll \exp \left\{O\left(\frac{\sqrt{\log x}}{\log _{2} x}\right)\right\},
$$

on obtient de $(2 \cdot 12),(2 \cdot 13),(2 \cdot 15)$ l'inégalité

$$
\left\|A_{x}\right\|_{1} \leq \sqrt{x}\left\|A_{x}\right\|_{\infty} \exp \left\{(\max \{-1 /(2 \vartheta),-\vartheta / 4\}+o(1)) \sqrt{\log x \log _{2} x}\right\} .
$$

Le choix optimal $\vartheta=\sqrt{2}$ fournit le résultat recherché.

Dans l'exemple développé pour établir le Lemme 2.1 on a

$$
\left\|A_{x}\right\|_{2}=\left\|A_{x}\right\|_{1} / \sqrt{\Psi(x, y)} .
$$

L'inégalité $(2 \cdot 14)$ fournit donc

$$
\left\|A_{x}\right\|_{1} /\left\|A_{x}\right\|_{\infty} \leq \sqrt{\Psi(x, y)}=\sqrt{x} \exp \left\{-\frac{\sqrt{2}}{4} \sqrt{\log x \log _{2} x}\right\},
$$

et on retrouve ainsi immédiatement pour cette famille d'exemples particuliers la majoration du Lemme 2.2.

Conclusion. Pour espérer reserrer l'encadrement de $S\left(\Lambda_{x}\right)$, il nous semble important de connaître le sous-ensemble de $\Gamma^{\pi(x)}$ où $P_{x}(\mathbf{z})$ prend de grande valeur. Ainsi, si cet ensemble est de mesure petite, on pourrait améliorer la majoration $(2 \cdot 14)$ et par conséquent le Lemme 2.2. En revanche, si cet ensemble est toujours de mesure relativement grande, la mesure de l'ensemble $I$ est plus grande que celle annoncée et alors la minoration du Lemme 2.1 peut être améliorée. Nous n'avons pas pu tirer profit d'une telle dichotomie de type heuristique. La minoration utilise un argument probabiliste, il est naturel de penser que c'est la majoration qu'il faille améliorer.

\section{Références}

[1] R. Balasubramanian, B. Calado and H. Queffélec, The Bohr inequality for ordinary Dirichlet series, Studia Math. 175 (2006), 285-304.

[2] M. Balazard, Remarques sur un théorème de G. Halász et A. Sárközy, Bull. Soc. Math. France 117 (1989), 389-413.

[3] J.-P. Kahane, Some Random Series of Functions, 2nd ed., Cambridge Univ. Press, Cambridge, 1985. 
[4] S. V. Konyagin and H. Queffélec, The translation $\frac{1}{2}$ in the theory of Dirichlet, Real Anal. Exchange 27 (2001/02), 155-175.

[5] H. Queffélec, H. Bohr's vision of ordinary Dirichlet series; old and new results, J. Analysis 3 (1995), 43-60.

[6] G. Tenenbaum, Introduction à la théorie analytique et probabiliste des nombres, 2nd ed., Cours Spécialisé, 1, Soc. Math. France, 1995.

Institut de Mathématiques de Jussieu

UMR 7586, Case 7012

Université Paris 7 - Denis Diderot

2, place Jussieu

F-75251 Paris Cedex 05, France

E-mail: breteche@math.jussieu.fr 